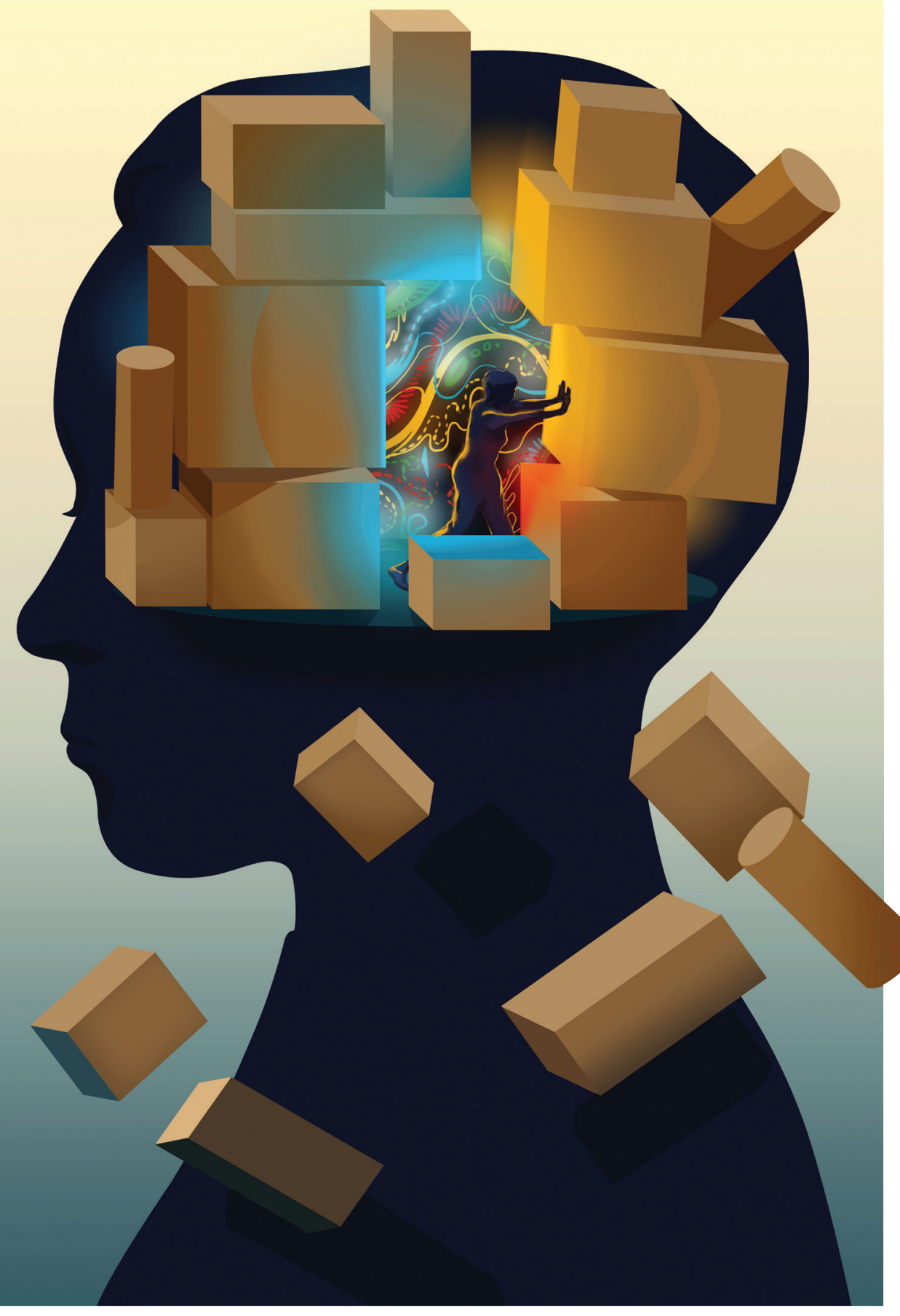

MEMORY

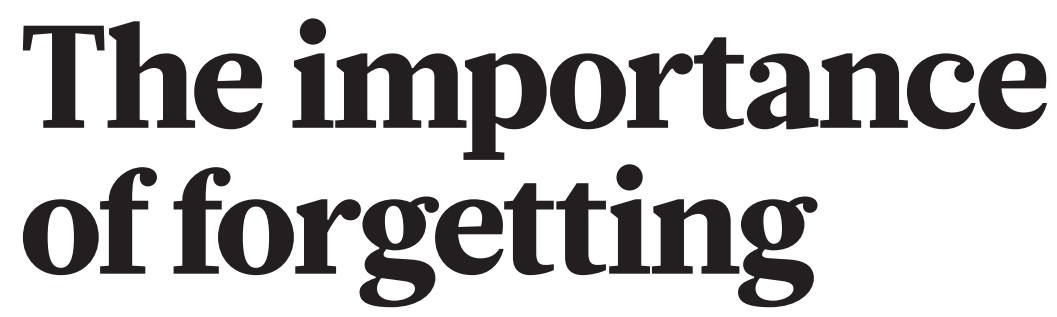

Long thought to be a glitch of memory, the ability to forget is actually crucial to how the brain works, researchers are realizing.
$\mathrm{M}$ emories make us who we are. They shape our understanding of the world and help us to predict what's coming. For more than a century, researchers have been working to understand how memories are formed and then fixed for recall in the days, weeks or even years that follow. But those scientists might have been looking at only half the picture. To understand how we remember, we must also understand how, and why, we forget.

Until about ten years ago, most researchers thought that forgetting was a passive process in which memories, unused, decay over time like a photograph left in the sunlight. But then a handful of researchers who were investigating memory began to bump up against findings that seemed to contradict that decades-old assumption. They began to put forward the radical idea that the brain is built to forget.

A growing body of work, cultivated in the past decade, suggests that the loss of memories is not a passive process. Rather, forgetting seems to be an active mechanism that is constantly at work in the brain. In some - perhaps even all - animals, the brain's standard state is not to remember, but to forget. And a better understanding of that state could lead to breakthroughs in treatments for conditions such as anxiety, post-traumatic stress disorder (PTSD), and even Alzheimer's disease.

"What is memory without forgetting?" asks Oliver Hardt, a cognitive psychologist studying the neurobiology of memory at

McGill University in Montreal, Canada. "It's impossible," he says. "To have proper memory function, you have to have forgetting."

\section{BIOLOGY OF FORGETTING}

Different types of memory are created and stored in varying ways, and in various areas of the brain. Researchers are still pinpointing the details, but they know that autobiographical memories - those of events experienced personally - begin to take lasting form in a part of the brain called the hippocampus, in the hours and days that follow the event. Neurons communicate with each other through synapses - junctions between these cells that include a tiny gap across which chemical messengers can be sent. Each neuron can be connected to thousands of others in this way. Through a process known as synaptic plasticity, neurons constantly produce new proteins to remodel parts of the synapse, such as the receptors for these chemicals, which enables the neurons to selectively strengthen their connections with one another. This creates a network of cells that, together, encode a memory. The more often a memory is recalled, the stronger its neural network becomes. Over time, and through consistent recall, the memory becomes encoded in both the hippocampus and the cortex. Eventually, it exists independently in the cortex, where it is put away for long-term storage. 
Neuroscientists often refer to this physical representation of a memory as an engram. They think that each engram has a number of synaptic connections, sometimes even in several areas of the brain, and that each neuron and synapse can be involved in multiple engrams.

Much is still unknown about how memories are created and accessed, and addressing such mysteries has consumed a lot of memory researchers' time. How the brain forgets, by comparison, has been largely overlooked. It's a remarkable oversight, says Michael Anderson, who studies cognitive neuroscience at the University of Cambridge, UK. "Every species that has a memory forgets. Full stop, without exception. It doesn't matter how simple the organism is: if they can acquire lessons of experience, the lessons can be lost," he says. "In light of that, I find it absolutely stunning that neurobiology has treated forgetting as an afterthought."

It wasn't at the forefront of Ron Davis's mind when he uncovered evidence of active forgetting in fruit flies (Drosophila melanogaster) in 2012. Davis, a neuroscientist at the Scripps Research Institute in Jupiter, Florida, was studying the intricacies of memory formation in the flies' mushroom bodies (dense networks of neurons in insect brains that store olfactory and other sensory memories). He was especially interested in understanding the influence of dopamine-producing neurons that connect with these structures. Dopamine, a neurotransmitter, is involved in moderating a host of behaviours in the fly brain, and Davis proposed that this chemical messenger might also play a part in memory.

Intriguingly, Davis found that dopamine is essential to forgetting ${ }^{1}$. He and his colleagues conditioned transgenic flies to associate electric shocks with certain odours, thereby training the insects to avoid them. They then activated the dopaminergic neurons and observed that the flies quickly forgot the association. Yet blocking the same neurons preserved the memory. "They were regulating how memories could be expressed," Davis says, essentially providing a 'forget' signal.

Further investigation, involving a technique that enabled the researchers to monitor the activity of neurons in living flies, demonstrated that these dopamine neurons are active for long periods, at least in flies. "The brain is always trying to forget the information it's already learnt," Davis says.

\section{FROM FLIES TO RODENTS}

A few years later, Hardt found something similar in rats. He was investigating what happens at the synapses of neurons that are involved in longterm memory storage. Researchers know that memories are encoded in the mammalian brain when the strength of the connection between neurons increases. That connection strength is determined by the amount of a particular type of receptor found at the synapse. Known

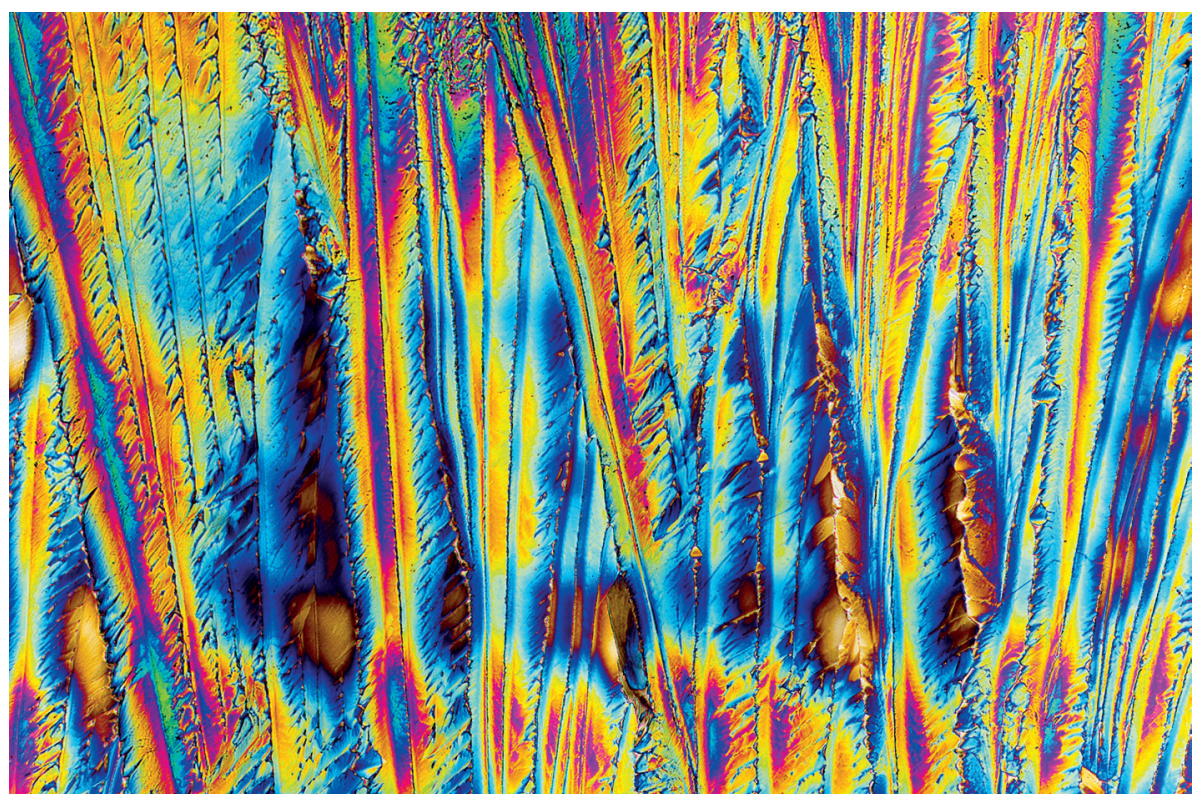

The neurotransmitter dopamine is now known to play an essential part in memory.

as AMPA receptors, the presence of these structures must be maintained for a memory to remain intact. "The problem," Hardt says, "is that none of these receptors are stable. They are moved in and out of the synapse constantly and turn over in hours or days."

Hardt's lab showed that a dedicated mechanism continuously promotes the expression of AMPA receptors at synapses. Yet some memories are still forgotten. Hardt proposed that AMPA receptors can also be removed, which suggests that forgetting is an active process. If that were true, then preventing the removal of AMPA receptors should prevent forgetting. When Hardt and his colleagues

The brain is always trying to forget the information. it's already learnt." blocked the mechanism behind AMPA-receptor removal in the hippocampi of rats, as expected, they found that the rats were prevented from forgetting the locations of objects ${ }^{2}$.

To forget certain things, it seemed that the rat brain had to proactively destroy connections at the synapse. Forgetting, Hardt says, "is not a failure of memory, but a function of it".

Paul Frankland, a neuroscientist at the Hospital for Sick Children in Toronto, Canada, had also found evidence that the brain is wired to forget. Frankland was studying the production of new neurons, or neurogenesis, in adult mice. The process had long been known to occur in the brains of young animals, but had been discovered in the hippocampi of mature animals only about 20 years earlier. Because the hippocampus is involved in memory formation, Frankland and his team wondered whether increasing neurogenesis in adult mice could help the rodents to remember.

In a paper published in 2014, the researchers found precisely the opposite: rather than making the animals' memories better, increasing neurogenesis caused the mice to forget more ${ }^{3}$. As contradictory as that initially seemed to Frankland, given the assumption that new neurons would mean more capacity for (and potentially better) memory, he says it now makes sense. "When neurons integrate into the adult hippocampus, they integrate into an existing, established circuitry. If you have information stored in that circuit and start rewiring it, then it's going to make that information harder to access," he explains.

Because the hippocampus is not where long-term memories are stored in the brain, its dynamic nature is not a flaw but a feature, Frankland says - something that evolved to aid learning. The environment is changing constantly and, to survive, animals must adapt to new situations. Allowing fresh information to overwrite the old helps them to achieve that.

\section{HUMAN NATURE}

Researchers think that the human brain might operate in a similar way. "Our ability to generalize new experiences is, at least in part, due to the fact that our brains engage in controlled forgetting," says Blake Richards, who studies neural circuits and machine learning at the University of Toronto Scarborough. Richards suggests that the brain's ability to forget might prevent an effect known as overfit: in the field of artificial intelligence, this is defined as when a mathematical model is so good at matching the data it has been programmed with that it is unable to predict which data might come next.

In a similar way, if a person were to remember every detail from an event such as a dog attack - that is, not just the sudden movement that scared the dog at the park, causing it to snarl and bite, but also the dog's floppy ears, the colour of its owner's T-shirt and the angle of the Sun - it might be more difficult for them to generalize across experiences to prevent themselves being bitten again in the future. "If you 


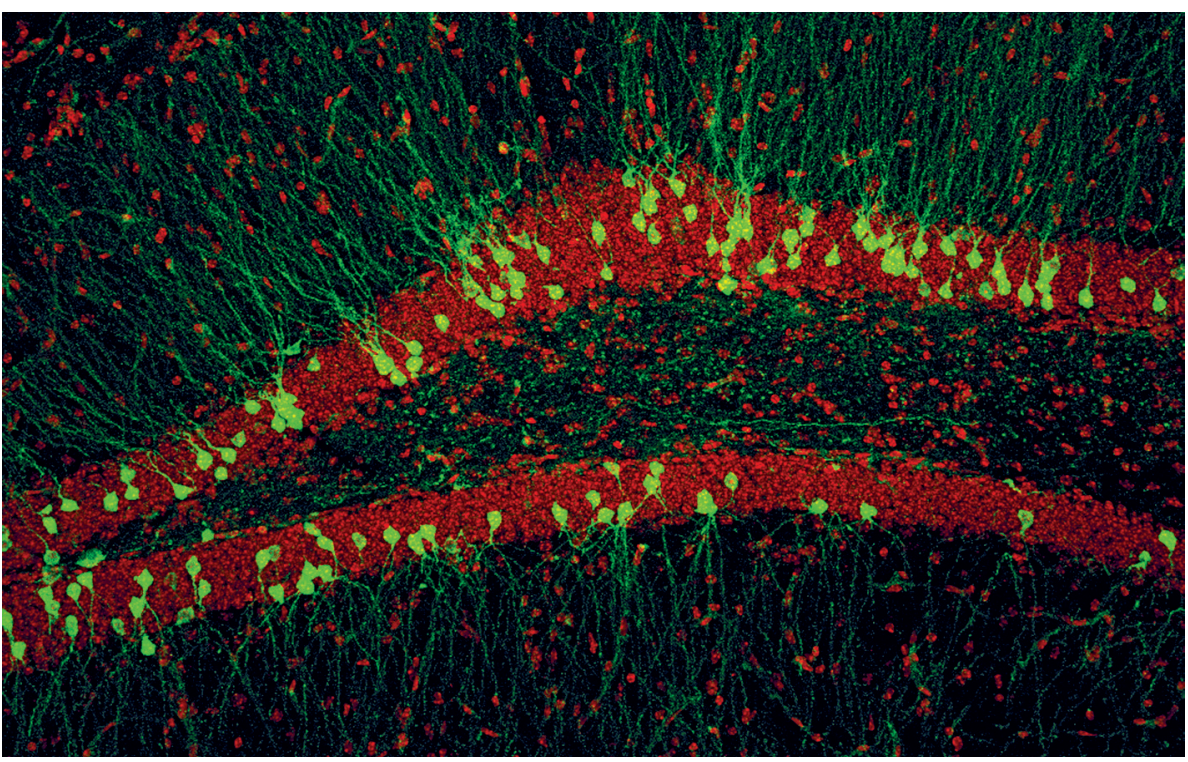

The integration of new neurons (green) into the hippocampus (red bands) degrades stored memories.

wash out a few details but retain the gist, it helps you to use it in novel situations," Richards says. "It's entirely possible that our brain engages in a bit of controlled forgetting in order to prevent us from overfitting to our experiences."

Studies of people with exceptional autobiographical memories or with impaired ones seem to bear this out. People with a condition known as highly superior autobiographical memory (HSAM) remember their lives in such incredible detail that they can describe the outfit that they were wearing on any particular day. But despite their exceptional ability to recall such information, these individuals tend not to be particularly accomplished and seem to have an increased tendency for obsessiveness, "which is exactly what you'd predict from someone who can't extract themselves from specific instances", says Brian Levine, a cognitive neuroscientist at the Rotman Research Institute at Baycrest Health Sciences in Toronto.

Those with severely deficient autobiographical memory (SDAM), however, are unable to vividly recall specific events in their lives. As a result, they also have trouble imagining what might happen in the future. Yet in Levine's experience, people with SDAM tend to do particularly well in jobs that require abstract thinking - probably because they are not weighed down by the nitty-gritty. "We think SDAM people, through a lifetime of practice of not having episodic memory, have an ability to cut across episodes," Levine says. "They're good at solving problems."

Research on forgetting in people without HSAM or SDAM is also beginning to show how important the process is for a healthy brain. Anderson's team has been digging deep into how active forgetting occurs in humans, using a combination of functional magnetic resonance imaging and magnetic-resonance spectroscopy to look at levels of the inhibitory neurotransmitter GABA ( $\gamma$-aminobutyric acid) in the hippocampus. By scanning participants who were attempting to quash certain thoughts, the researchers found that the higher someone's GABA levels were, the more a region of the brain called the prefrontal cortex suppressed their hippocampus, and the better they were at forgetting ${ }^{4}$."We were able to link successful forgetting to a particular neurotransmitter in the brain," Anderson says.

\section{TRYING TO FORGET}

By better understanding how we forget, through the lenses of both biology and cognitive psychology, Anderson and other researchers might be edging nearer to improving treatments for anxiety, PTSD and even Alzheimer's disease.

Anderson's work to measure GABA levels in the brain might indicate a mechanism that underpins the effectiveness of benzodiazepines - anti-anxiety drugs such as diazepam that have been prescribed since

\section{"Memory, have long known that} first and such medication works foremost, is by enhancing the functhere to serve tion of GABA receptors, anadaptive thereby helping to purpose." dampen anxiety, but they Anderson's findings offer an explanation: if the prefrontal cortex commands the hippocampus to inhibit a thought, the hippocampus can't respond unless it has enough GABA. "The prefrontal cortex is the general, sending commands from on high to suppress activity in the hippocampus," Anderson says. "If there aren't troops on the ground, those commands fall on deaf ears."

GABA's crucial role in suppressing unwanted thoughts also has implications for phobias, schizophrenia and depression. Various symptoms of these conditions - including flashbacks, obsessive thoughts, depressive rumination and difficulty controlling didn't understand why. thoughts - have been linked to an overactive hippocampus. "We think we have a key mechanistic framework that links together all of those different symptoms and disorders," Anderson says.

His group's research might also have implications for treating PTSD, a condition perceived to be a problem of remembering a traumatic episode too well, but one that, at its root, is really an issue of forgetting. A better understanding of how to help people make traumatic memories less intrusive could help researchers to treat some of the most intractable cases. When Anderson and his colleagues looked at what happens when volunteers suppress unwanted memories - a process he calls motivated forgetting - they found that people who reported more traumatic experiences were particularly good at repressing specific memories $^{5}$. Understanding the cognitive psychology that underlies that ability, as well as the mental resilience that is necessary for developing it, could help to improve treatment for PTSD.

Hardt thinks that Alzheimer's disease might also be better understood as a malfunction of forgetting rather than remembering. If forgetting is truly a well-regulated, innate part of the memory process, he says, it makes sense that dysregulation of that process could have negative effects. "What if what's actually going on is an overactive forgetting process that goes haywire and erases more than it should?" he asks.

That question is yet to be answered. But more memory researchers are shifting their focus to examine how the brain forgets, as well as how it remembers. “There's an increasing understanding that forgetting is a collection of processes in its own right, to be distinguished from encoding and consolidation and retrieval," Anderson says.

In the past decade, researchers have begun to view forgetting as an important part of a whole. "Why do we have memory at all? As humans, we entertain this fantasy that it's important to have autobiographical details," Hardt says. "And that's probably completely wrong. Memory, first and foremost, is there to serve an adaptive purpose. It endows us with knowledge about the world, and then updates that knowledge." Forgetting enables us as individuals, and as a species, to move forwards.

"Evolution has achieved a graceful balance between the virtues of remembering and the virtues of forgetting," Anderson says. "It's dedicated to both permanence and resilience, but also to getting rid of things that get in the way." -

Lauren Gravitz is a science journalist in San Diego, California.

1. Berry, J. A., Cervantes-Sandoval, I., Nicholas, E. P. \& Davis, R. L. Neuron 74, 530-542 (2012).

2. Migues, P. V. et al. J. Neurosci. 36, 3481-3494 (2016)

3. Akers, K. G. et al. Science 344, 598-602 (2014).

4. Schmitz, T. W., Correia, M. M., Ferreira, C. S., Prescot, A. P. \& Anderson, M. C. Nature Commun. 8, 1311 (2017)

5. Hulbert, J. C. \& Anderson, M. C. J. Exp. Psychol. Gen. 147, 1931-1949 (2018). 